\title{
Indications for lateral lymph node dissection in patients with rectal neuroendocrine tumors: A case report and review of the literature
}

\author{
YOSHIAKI FUJII $^{1}$, KENJI KOBAYASHI ${ }^{1}$, SYO KIMURA $^{1}$, SHUHEI UEHARA $^{1}$, \\ HIROTAKA MIYAI $^{1}$ and SHUJI TAKIGUCHI ${ }^{2}$
}

${ }^{1}$ Department of Surgery, Kariya Toyota General Hospital, Kariya, Aichi 448-0850; ${ }^{2}$ Department of

Gastroenterological Surgery, Nagoya City University Graduate School of Medical Sciences, Nagoya, Aichi 467-8602, Japan

Received July 16, 2020; Accepted February 5, 2021

DOI: $10.3892 / \mathrm{mco} .2021 .2242$

\begin{abstract}
The optimal surgical indications for small rectal neuroendocrine tumors (NETs) are controversial. Generally, treatment guidelines for rectal NETs $>2 \mathrm{~cm}$ or with potential lymph node (LN) metastasis recommend formal oncologic low anterior resection (LAR) with total mesorectal excision (TME). However, rectal NETs have the potential to metastasize to the lateral lymph nodes (LLNs). To the best of our knowledge, there are no detailed reports in English on LLN metastasis from rectal NETs. A 47-year-old man diagnosed with a rectal NET underwent endoscopic submucosal dissection (ESD). The pathological diagnosis was NET G1. The tumor was $10 \mathrm{~mm}$ in diameter, and the tumor depth reached the submucosal layer. A period of 3 years after ESD, the patient was diagnosed with $\mathrm{LN}$ metastasis in the mesorectum and LLN metastasis on the left side from the NET. Robotic TME and bilateral LN dissection were performed. The pathological findings indicated that two of the $18 \mathrm{LNs}$ in the mesorectum were metastatic, and all the LLNs on the left side were negative. In contrast, 1 of the 6 LLNs on the right side was metastatic. Early-stage rectal NETs can metastasize to the LLNs, and it is very difficult to detect LLN metastasis based on size alone. TME alone may be insufficient to treat rectal NETs, and additional LLN dissection may be an important treatment strategy. However, it is increasingly difficult to determine the surgical indications for optimally timed LLN dissection.
\end{abstract}

\section{Introduction}

Rectal neuroendocrine tumors (NETs) are relatively rare, with an annual incidence of 1.04 cases per 100,000 individuals (1). The incidence of rectal NETs has increased by almost ten-fold

Correspondence to: Dr Yoshiaki Fujii, Department of Surgery, Kariya Toyota General Hospital, 15-5-Sumiyoshicyou-Kariya-Aichi, Kariya, Aichi 448-0850, Japan

E-mail:kyabetu1208@yahoo.co.jp

Key words: rectum, neuroendocrine tumor, lateral lymph node dissection, metastasis over the past few decades, which is thought to be due to increased colorectal cancer screening, recent improvements in detection due to endoscopic developments, and a greater clinical understanding (2,3). Among rectal NETs, $93.3-100 \%$ are $1 \mathrm{~cm}$ or less at diagnosis (4). The World Health Organization (WHO) classified rectal NETs as low-grade malignant tumors; however, NETs and adenocarcinoma had similar survival if the tumor had lymph node (LN) metastasis or distant metastasis (5). Generally, treatment guidelines for rectal NETs larger than $2 \mathrm{~cm}$ or with potential LN metastasis recommend a formal oncologic low anterior resection (LAR) with total mesorectal excision (TME). However, rectal NETs in the lower rectum may metastasize to the lateral lymph nodes (LLNs) along alternate lymphatic passages outside of the mesorectal envelope, similar to adenocarcinoma in the lower rectum. Due to their low-grade malignant potential and very slow growth, metastatic LLNs are so small that preoperative identification with computed tomography (CT) and magnetic resonance imaging (MRI) may be difficult. There are no detailed reports in English about LLN metastasis from rectal NETs. Currently, the surgical indications for LLN metastasis from rectal NETs are unclear. Considering the lack of effective chemotherapy options, optimally timed radical resection may help improve the prognosis of rectal NETs.

\section{Case report}

A 47-year-old man underwent total colonoscopy as a routine health examination at another hospital 3 years ago. The examination revealed a hemispheric submucosal tumor $(10 \mathrm{~mm}$ in diameter) in the lower rectum that was located $7 \mathrm{~cm}$ from the anal verge at the anterior side of the rectal wall (Fig. 1A). The lesion did not exhibit a central depression or ulceration, and the pathological diagnosis was a NET. Additionally, no signs and symptoms of carcinoid syndrome were observed. Imaging examinations, including CT and MRI, were not performed at that time. He underwent endoscopic submucosal dissection (ESD) at that hospital, and the macroscopic findings of the resected specimen indicated that the primary tumor was $10 \mathrm{~mm}$ in diameter (Fig. 2A). The pathological tumor depth was limited to the submucosal layer (Fig. 2B), and lymphovascular 
invasion was detected. A pathological diagnosis of NET G1 was confirmed according to a $\mathrm{Ki}-67$ index of $1.6 \%$ (Fig. 2C). Immunohistochemical analysis for synaptophysin of the specimens revealed positive immunostaining of the tumor cells (Fig. 2D). The tumor margins were clear, and additional surgical resection was not performed at that time. A CT examination was performed three years after ESD and revealed LN swelling in the mesorectum and obturator space on the left side. The patient was referred to our hospital for surgery. Colonoscopy revealed a scar in the lower rectum after ESD, and the biopsy detected no evidence of local recurrence (Fig. 1B). Laboratory data revealed no abnormal findings. Contrast-enhanced CT images and T2-weighted MRI scans revealed 2 enlarged LNs (maximum size, $12.1 \mathrm{~mm}$ ) in the mesorectum and 5 enlarged LLNs (maximum size, $10 \mathrm{~mm}$ ) on the left side (Fig. 3A and B). LLNs were not detected on the right side. ${ }^{68} \mathrm{Ga}$-DOTATOC positron emission tomography $\left({ }^{68} \mathrm{Ga}\right.$-DOTATOC PET)/CT images revealed high ${ }^{68} \mathrm{Ga}$-DOTATOC uptake in the mesorectum and no abnormal uptake on either side of the obturator space (Fig. 3C) or any other distant organs. The patient was diagnosed with LN metastasis in the mesorectum and LLN metastasis on the left side from the NET.

Robotic TME and bilateral LN dissection were performed. The proximal LNs were dissected around the root of the inferior mesenteric artery (IMA). In the pelvic space, TME was performed up to the anal canal. Lateral lymphadenectomy was subsequently performed on both sides as follows. The ureter and hypogastric nerve were elevated, and the internal iliac vessels were subsequently cleared from the lymphatic tissue at a safe distance from the lateral side of the pelvic plexus. The LNs and fatty tissue were dissected from the obturator space. During the dissection, the obturator nerve was preserved. Following completion of the bilateral LN dissection, only the external vessels, internal iliac vessels and their branches, the obturator nerves, and the pelvic plexus remained. The operative time was $576 \mathrm{~min}$, and the intraoperative blood loss volume was $900 \mathrm{ml}$. The patient recovered well from surgery. He was discharged on postoperative day 7 , and adjuvant therapy was not performed. His defecation function was good, with no fecal incontinence. His voiding and sexual functions were preserved. The macroscopic and microscopic findings of the resected specimen indicated that there was no residual tumor in the rectum. Two of the $18 \mathrm{LNs}$ in the mesorectum contained metastases from the NET, and the LLNs on the left side (which included $13 \mathrm{LNs}$ ) were all negative. In contrast, one of the 6 LLNs on the right side contained metastasis from the NET. The patient was followed up with chest and abdominal CT every 6 months. At the one-year follow-up, no local recurrence and distant metastasis had been found.

\section{Discussion}

Rectal NETs are relatively rare. Due to increased screening with colonoscopy, the incidence of rectal NETs has increased in the past few years. In the National Comprehensive Cancer Network guidelines, rectal NETs $>2 \mathrm{~cm}$ with invasion into the muscularis propria or LN metastases should be treated with LAR (6). In the European Neuroendocrine Tumor Society guidelines and the North American Neuroendocrine Tumor Society consensus guidelines, patients with rectal NETs $>2 \mathrm{~cm}$ and 1- to 2-cm NETs with muscular invasion or positive LNs are recommended to undergo radical resection with $\mathrm{LN}$ dissection $(7,8)$. On the other hand, the surgical indications for rectal NETs $\leq 1 \mathrm{~cm}$ are still unclear. The incidences of LN metastasis in tumors of various sizes are 1.0\% ( $\leq 5 \mathrm{~mm}), 8.4 \%(6-10 \mathrm{~mm})$, $54.5 \%(11-20 \mathrm{~mm})$ and $66.7 \%(\geq 21 \mathrm{~mm})$. Based on the depth of invasion, the incidences of $\mathrm{LN}$ metastasis were $11.7 \%$ (limited to the mucosa or submucosa) and $87.5 \%$ (into or through the muscularis propria) (9). In our case, the tumor was $10 \mathrm{~mm}$ in diameter, and the pathological tumor depth was limited to the submucosal layer. There was no lymphatic invasion, but venous invasion was observed. Additional surgical resection including TME might have been indicated after ESD.

The lymphatic tract of the lower rectum below the peritoneal reflection consists of two patterns: Along the IMA in the mesorectum and along the internal iliac artery to the lateral pelvic floor. Accordingly, not only TME but also LLN dissection on both sides have been performed in Japan for advanced lower rectal cancer to achieve better local control (10). For rectal NETs, the indications for LLN dissection and how this approach contributes to patient prognosis are still unclear. In our case, the patient was preoperatively diagnosed with LN metastasis in the mesorectum and in the lateral pelvic floor on the left side from a rectal NET. We performed robotic TME and bilateral LN dissection following the treatment strategy for rectal cancer. However, the 13 resected LLNs on the left side were all negative, and 1 of the 6 LLNs on the right side was metastatic. With preoperative imaging examinations, including CT and MRI, we detected 5 enlarged LLNs on the left side. However, we did not detect LLNs on the right side. Pathological specimens, which were harvested from the lateral pelvic space, showed 13 negative LNs on the left side. In contrast, there was a metastatic $\mathrm{LN}(7 \mathrm{~mm})$ and 5 negative LNs on the right side (Fig. 4A). A pathological diagnosis of LLN metastasis was confirmed according to a Ki-67 index of $2 \%$ (Fig. 4C). Immunohistochemical analysis for synaptophysin and chromogranin $\mathrm{A}$ of the specimens revealed positive immunostaining of the tumor cells (Fig. 4B and D). Ushigome et al reported that $66 \%$ of patients who had LLN metastasis from a rectal NET had no metastatic LNs in the mesorectum. In this study, LLN dissection was performed for patients with enlarged LLNs $>7 \mathrm{~mm}$ on preoperative CT or MRI (11). Tables I and II show 12 reported cases of LLN metastasis from rectal NETs (12-22). Synchronous resection of primary rectal NETs and metastatic LLNs was performed in 8 cases (Table I), and heterochronous resection was performed in 4 cases (Table II). Seven patients showed no metastatic LNs in the mesorectum, and nine patients showed a primary tumor $\leq 2 \mathrm{~cm}$ in diameter. The tumor invasion depth was limited to the submucosa in 8 cases. Rectal NETs, even those with a small size and shallow depth of tumor invasion, can metastasize to the LLNs. Colorectal cancer develops from the mucosal epithelium; on the other hand, colorectal NETs develop from Kultschitzky cells that are located in the deep mucosa (23). The difference in origin may contribute to the slow metastasis to LLNs in the early stage. The recurrence interval is relatively long (18-276 m), and patients who undergo radical resection are expected to achieve long-term survival. Patients with rectal NETs should be followed up with imaging examinations over an extended period of time. Considering the characteristics of 
A
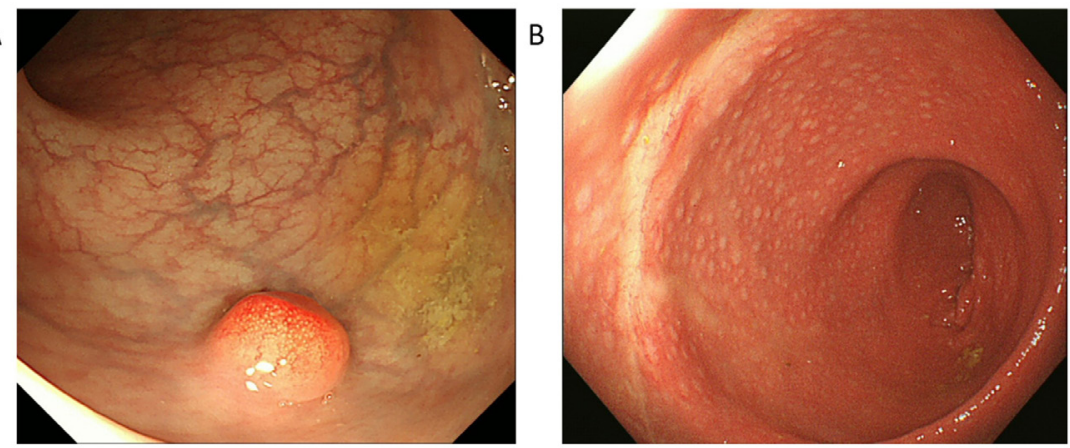

Figure 1. (A) Colonoscopy revealed a hemispheric submucosal tumor that was $7 \mathrm{~mm}$ in diameter in the lower rectum without central depression or ulceration. (B) Colonoscopy revealed a scar in the lower rectum after ESD, and the biopsy detected no evidence of local recurrence. ESD, endoscopic submucosal dissection.
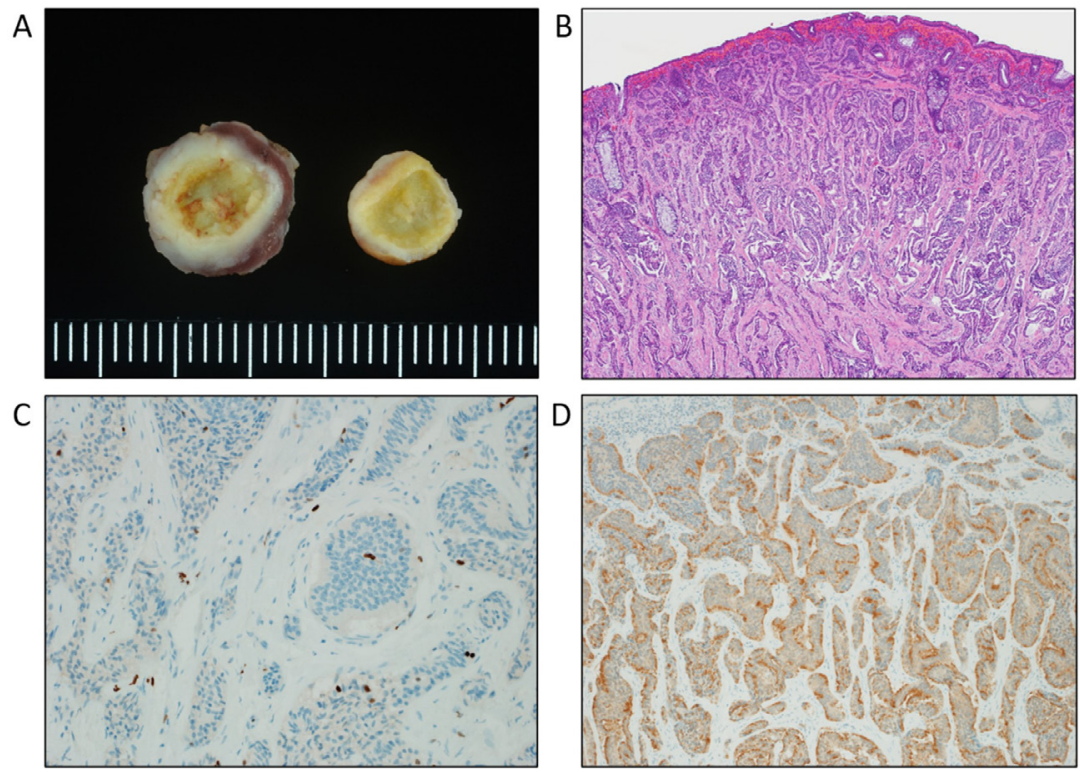

Figure 2. Histopathological findings of the resected specimen (primary tumor). (A) Macroscopic findings of the resected specimen indicated that the primary tumor was $10 \mathrm{~mm}$ in diameter. (B) Hematoxylin and eosin staining showed that the tumor cells spread in a rosette-like pattern (magnification, $\mathrm{x} 4)$. (C) The $\mathrm{Ki}-67$ index was $1.7 \%$ (magnification, $\mathrm{x} 400$ ). (D) Immunohistochemical staining for synaptophysin was positive (magnification, $\mathrm{x} 400$ ).
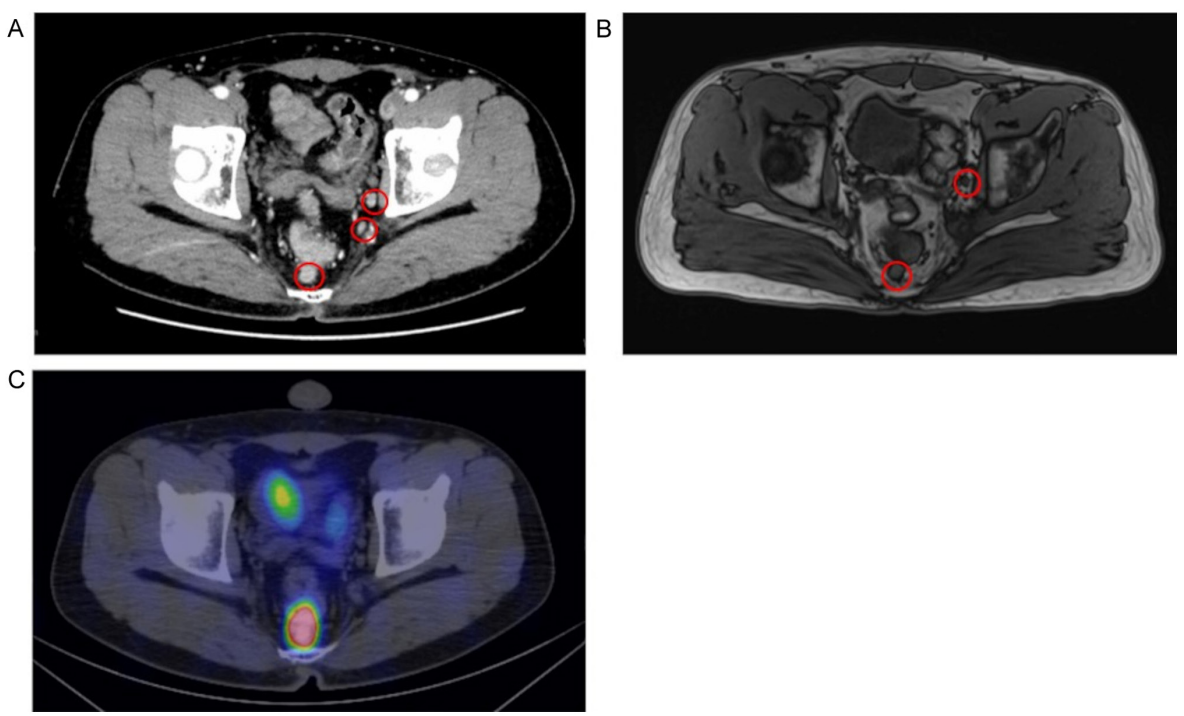

Figure 3. Preoperative imaging examinations performed 3 years after ESD. (A) Contrast-enhanced CT and (B) T2-weighted axial MRI revealed lymph node enlargement in the mesorectum and on the left side of the lateral pelvic space (red circle). The maximum lymph node size in the mesorectum was $12.1 \mathrm{~mm}$ and that in the left obturator space was $10 \mathrm{~mm}$. (C) ${ }^{68} \mathrm{Ga}$-DOTATOC PET imaging revealed high ${ }^{68} \mathrm{Ga}$-DOTATOC uptake in the mesorectum and no uptake in either side of the lateral pelvic space. ESD, endoscopic submucosal dissection. 

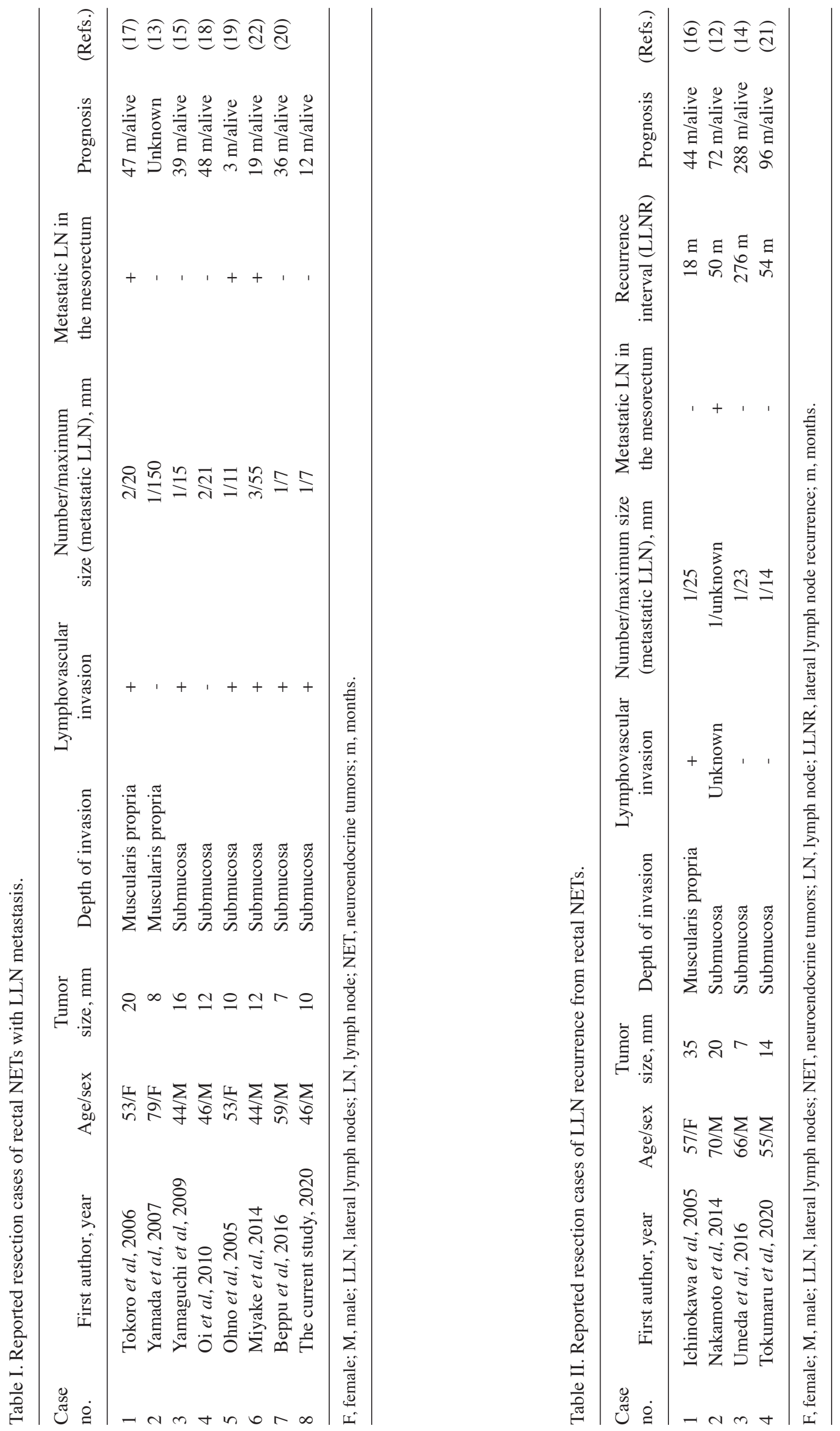

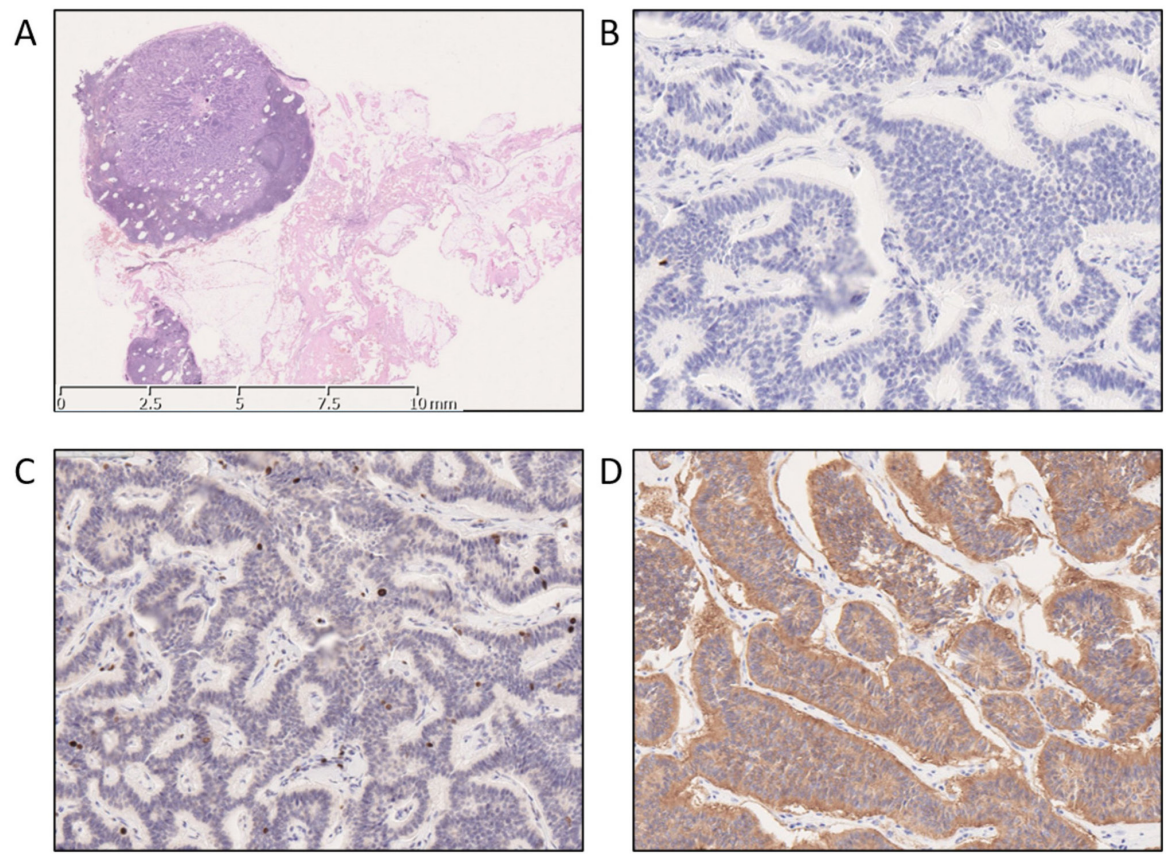

Figure 4. Histopathological findings of the resected specimen (metastatic LLN on the right side). (A) Hematoxylin and eosin staining showed that the diameter of the metastatic LLN on the right side was $7 \mathrm{~mm}$. (B) Immunohistochemical staining for chromogranin A was negative (magnification, $\mathrm{x} 400$ ). (C) The Ki-67 index was 2.0\% (magnification, $\mathrm{x} 400$ ). (D) Immunohistochemical staining for synaptophysin was positive (magnification, $\mathrm{x} 400$ ). LLN, lateral lymph nodes.

low-grade malignant potential and very slow growth of these tumors, watchful observation without LLN dissection may be an option for rectal NETs. However, if complete surgical resection is possible, LLN dissection may be an important treatment option.

In this case, there were more LLNs on the left side, and they were larger than those on the right side in the preoperative examinations; additionally, we diagnosed LLN metastasis on the left side. However, a metastatic LLN was present on the right side. On ${ }^{68} \mathrm{Ga}$-DOTATOC PET-CT, metastatic LLNs could not be detected. It may be difficult to diagnose LLN metastasis from rectal NETs with imaging examinations based on size alone. No evidence-based data on the surgical indications for LLN metastasis have been published to date. Despite the small sample size, these findings suggest that radical resection may be effective for improving prognosis due to the spread of rectal NETs, their slow growth, their low malignant potential and a lack of effective chemotherapy options. Rectal NETs are a relatively rare malignant tumor. A larger sample size and longer observation period may help establish an optimal treatment strategy for rectal NETs and LLN metastasis. This case study presented a case of a rectal NET that metastasized to the LLN. Early-stage rectal NETs can metastasize to the LLNs despite the characteristics of slow growth and low malignant potential. It is difficult to detect metastatic LLNs with preoperative imaging examinations based on size alone due to the aforementioned characteristics. However, radical resection, including resection of the metastatic LLNs, may contribute to a better prognosis, as suggested by the reported cases. It is exceedingly difficult to determine the surgical indications for optimally timed LLN dissection. We should keep several options in mind when planning a treatment strategy for rectal NETs.

\section{Acknowledgements}

Not applicable.

\section{Funding}

No funding was received.

\section{Availability of data and materials}

The datasets used and/or analyzed during the current study are available from the corresponding author on reasonable request.

\section{Authors' contributions}

YF collected the patient's clinical data and wrote the manuscript. KK, SK, SU and HM performed the surgery and postoperative management. ST made substantial contributions to conception and design, and revised the manuscript critically. YF and KK are responsible for confirming the authenticity of the raw data. All authors read and approved the final manuscript.

\section{Ethics approval and consent to participate}

The Ethics Committee of Kariya Toyota General Hospital provided approval for the current study due to the written informed consent obtained (approval no. 642).

\section{Patient consent for publication}

The patient provided written informed consent for the publication of the case details and any associated images. 


\section{Competing interests}

The authors declare that they have no competing interests.

\section{References}

1. Dasari A, Shen C, Halperin D, Zhao B, Zhou S, Xu Y, Shih T and Yao JC: Trends in the incidence, prevalence, and survival outcomes in patients with neuroendocrine tumors in the United States. JAMA Oncol 3: 1335-1342, 2017.

2. Fraenkel M, Kim M, Faggiano A, de Herder WW and Valk GD; Knowledge NETwork: Incidence of Gastroenteropancreatic neuroendocrine tumours: A systematic review of the literature. Endocr Relat Cancer 21: R153-R163, 2014.

3. Hallet J, Law CH, Cukier M, Saskin R, Liu N and Singh S: Exploring the rising incidence of neuroendocrine tumors: A population-based analysis of epidemiology, metastatic presentation, and outcomes. Cancer 121: 589-597, 2015.

4. Scherübl H: Rectal carcinoids are on the rise: Early detection by screening endoscopy. Endoscopy 41: 162-165, 2017.

5. Konishi T, Watanabe T, Kishimoto J, Kotake K, Muto T and Nagawa H; Japanese Society for Cancer of the Colon and Rectum: Prognosis and risk factors of metastasis in colorectal carcinoids: Results of a nationwide registry over 15 years. Gut 56: 863-868, 2007.

6. Kulke MH, Shah MH, Benson AB III, Bergsland E, Berlin JD, Blaszkowsky LS, Emerson L, Engstrom PF, Fanta P, Giordano T, et al: Neuroendocrine tumors, version 1.2015. J Nat Comp Cancer Net 13: 78-108, 2015.

7. Ramage JK, De Herder WW, Delle Fave G, Ferolla P, Ferone D, Ito T, Ruszniewski P, Sundin A, Weber W, Zheng-Pei Z, et al: ENETS consensus guidelines update for colorectal neuroendocrine neoplasms. Neuroendocrinology 103: 139-143, 2016.

8. Anthony LB, Strosberg JR, Klimstra DS, Maples WJ, O'Dorisio TM, Warner RR, Wiseman GA, Benson AB III and Pommier RF; North American Neuroendocrine Tumor Society (NANETS): The NANETS consensus guidelines for the diagnosis and management of gastrointestinal neuroendocrine tumors (nets): Well-differentiated nets of the distal colon and rectum. Pancreas 39: 767-774, 2010.

9. Kasuga A, Chino A, Uragami N, Kishihara T, Igarashi M, Fujita R, Yamamoto N, Ueno M, Oya M and Muto T: Treatment strategy for rectal carcinoids: A clinicopathological analysis of 229 cases at a single cancer institution. J Gastroenterol Hepatol 27: 1801-1807, 2012.

10. Hashiguchi Y, Muro K, Saito Y, Ito Y, Ajioka Y, Hamaguchi T, Hasegawa $\mathrm{K}$, Hotta $\mathrm{K}$, Ishida $\mathrm{H}$, Ishiguro $\mathrm{M}$, et al: Japanese society for cancer of the colon and rectum (JSCCR) guidelines 2019 for the treatment of colorectal cancer. Int J Clin Oncol 25 : $1-42,2020$

11. Ushigome H, Fukunaga $Y$, Nagasaki T, Akiyoshi T, Konishi T, Fujimoto Y, Nagayama S and Ueno M: Difficulty of predicting lymph node metastasis on CT in patients with rectal neuroendocrine tumors. PLoS One 14: e0211675, 2019.
12. Nakamoto T, Koyama U, Nakagawa T, Nakamura S, Ueda T, Nishigori N, Inoue T, Kawasaki K, Obara S, Fujii H and Nakajima Y: Four resections of metachronous liver metastases and lateral lymph node metastases of a rectal carcinoid tumor-a case report. Jpn J Cancer Chemother 41: 1829-1831, 2014 (In Japanese).

13. Yamada E, Mori A, Nagayama S, Okamoto T, Koyama T, Ito R and Onodera $\mathrm{H}$ : A case of a minute rectal carcinoid with a huge metastatic obturator lymph node. Jpn J Gastroenterol Surg 40: 491-496, 2007 (In Japanese).

14. Umeda S, Hishida M, Jinno S, Shimizu M, Kobayashi H, Nozaki $\mathrm{H}$ and Harada T: Lateral lymph node metastasis of rectal neuroendocrine tumor G1 23 years after transanal resection. Jpn J Gastroenterol Surg 49: 556-562, 2016 (In Japanese).

15. Yamaguchi K, Morita T, Okamura K, Kawamura T and Horita H: A case of rectal carcinoid tumor with a metastatic lymph node in the right lateral. Jpn Soc Coloproctol 62: 180-184, 2009 (In Japanese).

16. Ichinokawa M, Nakamura Y, Maeyama Y, Manase H, Taira K and Hishiyama H: A case of a rectal carcinoid tumor with solitary recurrence to the right lateral lymph node performed trans-sacral extirpation after systemic chemotherapy. J Jpn Surg Assoc 66: 3011-3014, 2005 (In Japanese).

17. Tokoro T, Okun K, Hida J, Ishimaru E, Ueda K, Yoshifuji T, Matsuzaki T, Minami Y and Shiozaki H: Radiofrequency ablation therapy for multiple liver metastases of rectal carcinoid-report of a case. Jpn J Gastroenterol Surg 39: 1816-1821, 2006 (In Japanese).

18. Oi K, Fukumoto Y, Nakamura S, Sawata $T$ and Shimizu T: Rectal carcinoid tumor, $12 \mathrm{~mm}$ in diameter, with metastasis to the internal iliac lymph nodes-a case report. J Jpn Surg Assoc 71: 2398-2401, 2010 (In Japanese).

19. Ohno R, Konshi T, Ueno M, Fukunaga Y, Nagayama S, Fujimoto Y, and Akiyoshi T: A rectal carcinoid tumor with lateral lymph node metastasis treated by laparoscopic total mesorectal excision with lateral lymph node dissection: A case report. Jpn J Endoscopic Surg 40: 200-213, 2005 (In Japanese).

20. Beppu N, Niki M, Kimura F, Matsubara N, Tomita N, Yanagi H and Yamanaka N: A case of rectal carcinoid, $7 \mathrm{~mm}$ in diameter, with skip metastasis to the lateral lymph node. Mol Clin Oncol 4: 549-552, 2016

21. Tokumaru Y, Matsuhashi N, Takahashi T, Imai H, Tanaka Y, Okumura N, Yamaguchi K and Yoshida K: Rectal neuroendocrine tumor developing lateral lymph node metastasis after curative resection: A case report. World J Surg Oncol 18: 74, 2020.

22. Miyake Y, Hasegawa J, Kim H, Mikata S, Shimizu J, Kim Y, Hirota M, Soma Y, Miwa H and Nezu R: A case of rectal carcinoid detected by nodal metastasis. Jpn J Gastroenterol Surg 47: 357-363, 2014 (In Japanese).

23. Marks $\mathrm{C}$ and Lamberty J: The cellular structure of bronchial carcinoids. Postgrad Med J 53: 360-363, 1977. International (CC BY-NC-ND 4.0) License. 